\title{
LE RÉCIT DANS LE DOCUMENTAIRE DE TYPE JOURNALISTIQUE
}

\author{
Christophe Deleu ${ }^{1}$
}

Comment le genre documentaire radiophonique de type journalistique s'est-il constitué, puis stabilisé à la radio ? Dans cet article, nous montrons comment la prise en compte de l'étude du récit permet d'interroger la notion de genre, ici le documentaire, en tenant compte de la situation de communication, ainsi que le permettent les emprunts aux recherches sur le récit en littérature. Nous analysons comment le genre documentaire de type journalistique à la radio, même s'il repose sur des formes de récits invariants, évolue néanmoins selon le contexte. Par exemple, la présence d'un médiateur-narrateur est constante depuis l'apparition du genre au milieu des années 1930. En revanche, sa place dans le dispositif a subi de profondes mutations (style de la narration, ton de la voix, rapport de places entre journalistes et interviewés...). Nous montrons ainsi comment ce type de productions médiatiques est mis en ondes à travers des récits, qui s'articulent autour d'un contrat d'information, qui perdure malgré la transformation des récits.

1 Christophe Deleu est maître de conférences (HDR) à l'Université de Strasbourg, SAGE, UMR 7363 et vice-président du GRER

Recherches en communication, $\mathrm{n}^{\circ} 37$ (2012). 
Le reportage est antérieur à la création de la radio. Il a été étudié par ceux qui se sont intéressés à l'émergence de la professionnalisation du journalisme en France. On citera par exemple les travaux de Ruellan (1993, p. 104) :

reportage signifiera : produit d'une démarche active de recherche et de divulgation d'informations réalisé par un individu placé en position (même provisoire) de témoin : reportage est donc à la fois une action et son résultat, être en reportage aboutit au produit. C'est une enquête et une écriture. Une démarche à deux pôles, et en deux espaces temps : un amont et un aval.

En France, le reportage s'est d'abord appelé « radioreportage ». Mais comme le remarque Méadel (1994, p. 276), à l'origine, il y a le terme de « reporter », utilisé en français dès le début du dix-neuvième siècle. Le terme « reportage » est souvent cité comme genre voisin quand on évoque le documentaire. Il nous semble donc important de relater l'apparition du genre. Nous empruntons à l'approche pragmatique qui s'appuie sur l'utilisation des terminologies des professionnels, en reprenant le terme de « reportage ». Mais nous optons aussi pour une taxinomie qui nous est propre, en définissant le « reportage » comme un « documentaire de type journalistique ». Il ne s'agit pas d'un choix arbitraire, le reportage journalistique a parfois été qualifié de « documentaire » par les professionnels des médias. Et nous expliquerons pourquoi les deux termes se sont parfois concurrencés. Par « documentaire radiophonique ", nous entendons : dispositif à caractère didactique, informatif et (ou) créatif, présentant des documents authentiques, qui suppose l'enregistrement de sons, une sélection de ceux-ci opérée par un travail de montage, leur agencement selon une construction déterminée, leur mise en ondes définitive effectuée par un travail de mixage, selon une réalisation préétablie, dans des conditions qui ne sont pas celles du direct ou du faux direct. Le terme « reportage » va prendre plusieurs sens à la radio, média du direct. Pour Sudre (1945, p. 86), le radioreportage est à l'origine « la description verbale faite sur place d'un événement ». Mais ce genre va subir plusieurs transformations importantes, à tel point qu'il englobe aujourd'hui plusieurs significations.

En 1923, les reportages sont réalisés en semi-différé. Méadel (1993, p. 279) rappelle ainsi que 
Le reporter téléphone son texte à une secrétaire qui le transcrit et le remet au speaker qui le lit. Ainsi fut diffusé en mai 1923 le reportage du match Carpentier-Niles par Edmond Dehorter sur le poste Radiola.

Mais très vite, le direct fait son apparition, et le reporter peut s'exprimer lui-même au micro. C'est donc la voix elle-même du journaliste que l'auditeur entend grâce au média de l'immédiateté. On emploierait aujourd'hui une autre terminologie : « en direct », " sur place »; ou « plateau de situation » pour la télévision. Les premiers radioreportages, qui concernent le secteur sportif, sont donc réalisés par Edmond Dehorter, surnommé « le parleur inconnu ». Ceux-ci vont ensuite s'élargir à d'autres thématiques comme les cérémonies officielles. Méadel (1994, p. 271) date de 1926-27 les premières techniques de radioreportage qui permettent de réaliser des émissions à l'extérieur, grâce aux lignes téléphoniques.

Plus tard, apparaît le « reportage à retardement » ou « reportage différé »(Sudre, 1945, p. 90). Pour Prot (1997, p. 511), le reportage peut donc aussi « faire l'objet d'un montage, c'est-à-dire d'un choix des meilleures séquences, d'un rapprochement avec d'autres éléments sonores (archives, illustrations musicales, etc.) qui viennent enrichir le document [...] ». Le reporter Jean Antoine va populariser cette forme radiophonique. Dès 1929, il fait financer ses radioreportages du Tour de France cycliste, diffusés à la radio d'État, par le journal L'Intransigeant. Dès 1932, Jean Antoine dispose du système d'enregistrement Marex, qui permet la diffusion des reportages enregistrés. Avant cette date, seules des émissions en direct étaient possibles hors des studios (Duval, 1979, p. 86). À partir de 1935, de nombreuses stations de radio ont des cars d'enregistrement qui leur permettent de réaliser ces reportages en différé. Il est important de rappeler que les conditions techniques pour réaliser des radioreportages ne sont pas celles d'aujourd'hui : les liaisons téléphoniques ne sont pas faciles à établir, le matériel n'est pas aisé à transporter, les perturbations sonores sont nombreuses. En revanche, la période est riche en inventions et prouesses techniques, même si l'émergence de projets semble aller plus vite que la possibilité de les réaliser. 
Le genre « radioreportage » ou « reportage » intègre le genre « interview ». Au départ, les deux genres, s'ils peuvent s'interpénétrer, sont indépendants l'un de l'autre : il peut y avoir une interview sans reportage (un entretien en studio), ou un reportage sans interview (la voix du journaliste et des sons d'ambiance). Mais aujourd'hui, le terme « reportage " suppose que l'auditeur puisse entendre une ou plusieurs personnes interviewées.

Comme le genre « interview », le genre « reportage » peut être réalisé par de nombreux professionnels de la radio. On songe en premier lieu aux journalistes, mais des speakers en seront aussi les auteurs, et même des écrivains. Aujourd'hui, à la radio, le terme désigne exclusivement des modules produits par des journalistes. Ces modules sont en général courts (ils n'excèdent pas deux minutes), présentent un « angle » (ou sujet) très resserré. Par exception, on peut entendre des reportages plus longs. Contrairement à l'interview (ou l'entretien) dont se sont emparés de nombreux professionnels de la radio, le reportage est donc avant tout une pratique réservée à une profession, dans le cadre d'espaces bien identifiés par l'auditeur (les journaux d'information, diffusés en général à heure fixe).

\section{L'omniprésence de la voix du journaliste}

À l'origine, dans les années 1920, le radioreportage, l'ancêtre du documentaire de type journalistique, n'est composé que d'un seul type de son : la voix du reporter qui commente ce qu'il voit. Ce n'est que plus tard qu'il ajoutera à son commentaire des interviews et des sons d'ambiance enregistrés sur place. Le journaliste occupe alors un rôle central, et va toujours rester présent malgré son évolution.

En termes d'évaluation professionnelle de ce type de production médiatique, on peut avancer que la qualité d'un radioreportage dépend donc beaucoup des capacités expressives de celui qui s'adresse à l'auditeur. Méadel (1994, p. 276) fixe comme critère d'embauche des radioreporters « les exigences sur la voix dont la qualité sonore sera rapidement vérifiée [...], sur la manière d'interviewer en direct et de faire parler [...]». Méadel (1994, p. 277) remarque aussi que « la médiation du reporter reste toujours première, la tranche de vie n'a de sens que commentée, montée, bref mise en scène ». Dans les années 1930, les auditeurs reconnaissent ainsi les reporters (Carlos Larronde, Jean 
Antoine, Alex Virot), qui créent leur propre syndicat en raison de leurs difficultés à s'intégrer au Syndicat des journalistes et de la profession, dominé par les journalistes de la presse écrite (Martin, 2005, p. 103).

C'est dans ce contexte de rivalité entre média de presse écrite et média radiophonique que les radioreporters forgent leur identité professionnelle. Faire entendre sa voix dans le reportage permet de se faire connaître du grand public, et d'être identifié. L'histoire du radioreportage est donc marquée par l'émergence de ces voix sur le terrain. C'est le journalisme sportif qui inaugure le genre, mais très vite d'autres secteurs sont l'objet de reportages. Martin (Id.) considère ainsi que 1935, année de la traversée d'un nouveau paquebot, le Normandie, et de la guerre déclenchée par Mussolini pour conquérir l'Éthiopie, constitue un tournant majeur :

L'année où, pour la première fois, à l'occasion d'événements retentissants, des reporters de radio quittent l'hexagone et le continent européen, et partent au loin, suivant l'exemple de leur confrères de presse écrite, avec la volonté de les égaler, voire de leur faire pièce. On peut parler alors sans aucune réserve de l'apparition de "grand reportage" à la radio.

Analyser l'évolution du radioreportage, c'est donc retracer l'émergence d'un champ, celui des journalistes de radio, qui souhaitent, à travers lui, marquer leur territoire, et établir leur légitimité en codifiant un genre. L'affirmation d'une identité professionnelle va s'exprimer à travers l'expulsion progressive de deux autres professions qui sont à l'origine associées au radioreportage, les speakers et les écrivains. C'est cette quête de légitimité professionnelle qui explique la tribune publiée par un des journalistes spécialistes du radioreportage, Jean Antoine, qui condamne « la manie de faire appel à des amateurs, des littérateurs en rupture de copie, mais rarement à des journalistes $»^{1}$.

Les événements qui marquent la fin de la Seconde Guerre mondiale sont « couverts » par les reporters radios, qui conçoivent des radioreportages dont la durée peut aller jusqu'à près d'un quart d'heure. La fin de

1 « Ici Radio Cité » du 29 juillet 1937, repris dans «L'année 1937 à la radio », Cahiers d'Histoire de la Radiodiffusion n 96, 4 juin 2008, Paris, Comité d'Histoire de la Radiodiffusion. 
la Seconde Guerre mondiale équivaut à un âge d'or du radioreportage. La revue Radio 44 (puis 45, etc.), dès son premier numéro, complète les productions diffusées à l'antenne de reportages écrits et illustrés par des photographies ${ }^{1}$. Le reporter est présenté comme un professionnel héroïque qui prend tous les risques pour informer l'auditeur. Dans Radio 45, on apprend par exemple que Michel Droit, correspondant de la radiodiffusion française, a été blessé en Allemagne :

Il accomplissait son devoir professionnel. [...] Le public a parfois tendance, lorsqu'il entend nos reportages de guerre, à penser, à dire et même écrire que c'est du "chiqué", que le bruit de la bataille a été reconstitué en studio. Michel Droit administre la preuve que les choses ne se passent pas exactement comme on l'imagine, et il a payé de son sang pour que les auditeurs sachent que nous n'abusons pas de leur confiance ${ }^{2}$.

Radio 45 publie aussi un article, "Comment s'effectue un reportage de Guerre », pour glorifier le travail des reporters, dont la voiture est à « 50 mètres des Allemands $»^{3}$. La guerre terminée, c'est ensuite l'exploration de territoires sociaux qui remplace le champ de bataille. Radio 45 publie par exemple un article, "Voyage au centre de la terre », sur un radioreportage enregistré au fond de la mine. C'est le journaliste Georges Jouin, qui conçoit le reportage radiophonique et l'article qui en retrace sa conception dans la revue. Le récit est écrit à la première personne du singulier, et le lecteur n'ignore rien des prouesses physiques accomplies par le reporter pour faire son métier ( «J'ai rampé pendant quarante mètres pour joindre le chantier », "Et je suis allé me laver pendant deux heures avec le savon des mineurs dont l'effet est tout à fait relatif ${ }^{4} \gg$ ). Georges Jouin se forge de son métier de reporter une représentation fidèle à celle de l'imaginaire collectif, souvent mythifiée au cinéma, et qui consacre le reportage comme «genre roi » (Ruellan, 1993, p. 99).

La définition du radioreportage n'est pas précisément établie. Les professionnels, comme les auditeurs, utilisent ces termes sans forcé-

1 Radio 44, $\mathrm{n}^{\circ} 1$.

2 Radio $45, \mathrm{n}^{\circ} 29$, p. 3

3 Radio $45, \mathrm{n}^{\circ} 13$, p. 3

4 Radio $45, n^{\circ} 44$, p. 5. 
ment entendre les mêmes sons. Comme illustration de cette ambiguité, on peut citer cet éditorial de Jean Guignebert, « À propos d'un radioreportage ", publié dans Radio $46^{1}$ et dans lequel il doit répondre à un auditeur, qui a reproché à un radioreporter d'avoir limité son intervention devant le micro à un monologue sans élément sonore. Jean Guignebert distingue ce type de monologues, qui ne relève pas selon lui du radioreportage, des véritables radioreportages sonores.

Cette ambiguïté taxinomique sur la définition du radioreportage va néanmoins perdurer. Dans Le Monde comme il va, production du Journal parlé de la Radiodiffusion française (1954-1957), coexistent des émissions dans lesquelles sont juxtaposées des séquences enregistrées sur le terrain, et commentées par la voix de l'instance médiatique (par exemple l'émission du 28 janvier 1954), et d'autres qui reposent exclusivement sur la voix du reporter (comme l'émission du 8 juin 1953). Cette dernière émission, d'une demi-heure, n'est composée que d'un récit de voyage, ponctué de quelques extraits de musique. Le journaliste est encore ce médiateur, qui raconte un événement exclusivement par sa voix.

La fonction du reporter va évoluer. Dans le documentaire journalistique d'après-guerre, le reporter décrit toujours ce qu'il voit, mais il n'est plus seulement dans une perspective de traitement de l'actualité en direct, «à chaud». S'il conserve sa place dans le dispositif, c'est davantage pour " raconter » d'une manière plus distante et posée que dans le reportage d'actualité d'avant-guerre.

C'est à ce moment-là que le reportage va désigner, au sein des rédactions de radio, des productions de natures différentes : le reportage comme module d'assez courte durée, enregistré et intégré dans les journaux d'actualité, le reportage comme intervention d'un journaliste en direct, toujours au sein d'un journal radiophonique, et le reportage comme format plus long (une demi-heure environ), diffusé en dehors des journaux d'actualité, selon une périodicité régulière. Il ne s'agit plus ici de traiter l'actualité sous forme événementielle, mais de prendre du recul pour appréhender le réel d'une autre manière. Si le terme « documentaire » apparait, après la Seconde Guerre mondiale, dans les formats

1 Radio 46, n 79, p. 3. 
longs radiophoniques qui dépendent du secteur de l'information, c'est en partie pour distinguer le reportage court du reportage long à la radio, et rapprocher ce dernier du documentaire cinématographique. Peu à peu, néanmoins, la notion de « documentaire » va tomber en désuétude chez les journalistes de radio, qui vont continuer à lui préférer le terme de « reportage », quitte à ce que cela introduise une confusion entre les différents dispositifs qu'une telle notion recoupe. Aujourd'hui, c'est le format long lui-même qui est tombé en désuétude dans le secteur de l'information, où les formes courtes se sont imposées. Pour la France, Interception, diffusée sur France Inter depuis 1999, constitue une des rares exceptions. Il n'y a pas de reportage long sur les radios généralistes privées (RTL, Europe1, RMC).

\section{La permanence du genre interview}

Dès que les techniques de transmission puis d'enregistrement du son l'ont permis, le reporter a ajouté à sa voix d'autres éléments au reportage - interviews, sons d'ambiance - afin de rapprocher l'auditeur de l'événement traité. Certes, pour la période des années 1920 et 30, nous manquons d'archives sonores pour retracer l'évolution du radioreportage, mais la description des programmes dans la presse magazine permet en partie de palier cette absence. Dès le milieu des années 1930, certains radioreportages préfigurent le documentaire d'aujourd'hui, et la radio privée Radio Cité, qu'ont rejointe deux des reporters radio les plus célèbres, Carlos Larronde et Jean Antoine, fait figure de précurseur. Dans Radio Magazine du 26 avril 1936, on peut ainsi lire que

Le car de radioreportage de l'Intransigeant (partenaire de Radio Cité) était à Londres. M. Michel Ferry en a profité pour glaner les plus suggestifs croquis sonores de la capitale anglaise. Un montage adroit a renforcé le caractère évocateur de ce panorama radiophonique. Rues de la cité, spectacle de la relève de la garde du château de Buckingham, prières de la capitale Saint-Paul, hymnes populaires dans les squares, crieurs de journaux hurlant des titres à sensation, chômeurs jouant de la musique dans les rues $[\ldots]$ : cette heure de vivants enregistrements, entrecoupés d'interviews prises sur le vif, a réuni les plus justes et les plus divers tableaux de la vie londonienne. Quelles ressources s'ouvrent aux informateurs [...]. Mais tout l'intérêt de la méthode est de nous faire connaître les propos et les sentiments de 
personnages inédits pour nous plus que celle d'un reporter qui "s'écoute parler"'.

Malheureusement, ce type d'articles, aussi descriptifs soient-ils, ne détaillent pas assez la construction des émissions, et l'on ne peut pas différencier ici ce qui relève de l'interview, de la séquence prise sur le vif, et du commentaire du journaliste.

Dans les émissions d'hier, comme dans celles d'aujourd'hui, l'instance médiatique signifie sa présence dans l'interview. Méadel (1994, p. 278) remarque que pour les reportages des années 1930, « les personnages sont introduits de manière formelle par des salutations (si aujourd'hui l'auditeur repère immédiatement ces reportages comme datés, c'est sans doute en grande partie à cause du formalisme de ces échanges) ». Mais par « interview », il ne faut guère ici entendre un entretien approfondi, mais plutôt un recueil de propos anecdotiques qui marquent la présence du reporter dans un lieu. L'interview n'est pas encore considérée comme un dispositif qui permet de révéler les sentiments des individus.

\section{L'évolution des conditions techniques}

Dans l'histoire des médias, l'évolution des techniques contribue aussi au développement voire à la création de nouvelles formes radiophoniques. Dans les années 1930 et 40, enregistrer à l'extérieur de la radio relève de la prouesse technique, tant la lourdeur du matériel handicape les déplacements des équipes de reporter. Dans la longue histoire du documentaire à la radio, mais aussi du cinéma et de la télévision, l'ingénieur polonais Stefan Kudelski joue un rôle majeur en inventant le Nagra en 1949, qui permet d'enregistrer du son sur bande magnétique, et donc d'éviter de devoir recourir à un camion-son pour faire un reportage. Prot (1997 : 338) rappelle que Kudelski rencontre de nombreuses difficultés pour faire connaître son matériel, les services des radios prévoyant que le Nagra révolutionne les conditions d'enregistrement, ce qu'ils perçoivent comme une menace pour l'emploi des techniciens. En 1952, Stefan Kudelski gagne le prix du concours international de l'enregistrement sonore organisé par Jean Thévenot avec un

1 Article de Pierre Domene, «La politique des ondes, un magazine sonore et parlant », Radio Magazine du dimanche 26 avril 1936, p. 3. 
documentaire sur le bourdon de Notre-Dame de Paris, et se fait ainsi connaître des professionnels de la radio. À partir de cette date, le Nagra apparaît dans toutes les radios, et rend les conditions d'enregistrement beaucoup plus souples. Europe 1, créée en 1954, assure la popularité de l'appareil d'enregistrement. Yann Paranthoën, l'un des auteurs de documentaires les plus connus, qui commence son parcours professionnel en 1957 dans le service public, en fera son outil de prédilection, et retracera son histoire dans l'émission On nagra, il enregistrera, en 1987 '. La miniaturisation des appareils d'enregistrement connaît un nouvel essor avec l'apparition du Minidisk, puis de l'Edirol. Plus léger, parfois invisible, ce matériel va permettre d'inventer de nouveaux dispositifs radiophoniques.

Dès la fin des années 1940, le documentaire de type journalistique nous semble relativement bien défini. Les contributions rassemblées dans l'unique numéro de La chambre d'Echo, éditée par le Club d'essai de la Radiodiffusion française en 1947, témoignent de la nature des enjeux pour le documentaire, puisque c'est à cette époque qu'on tente de distinguer le documentaire du reportage. Jacques Peuchmaurd, qui vient de concevoir son émission Paris-Brest, présentée comme un documentaire, affirme que

Dans le reportage considéré comme genre défini, le rôle du reporter est prépondérant. Il parle sur le fond sonore que l'oreille du micro enregistre, décrit le décor, commente les événements, ne s'efface que rarement devant l'élément sonore brut. [...] Dans le documentaire, au contraire, le reporter disparaît. Il n'est plus qu'un homme qui tient en main un micro : un homme muet. De même que la caméra ne prend que des images, le micro ne prend que des sons, des sons bruts sans commentaire. Ce n'est qu'ensuite, au montage en studio, qu'intervient la parole, comme elle apparaît au-dessus des images dans le documentaire cinématographique $^{2}$.

1 Diffusée dans le cadre de l'Atelier de création radiophonique, France Culture, 8 mars 1987, édition CD par Radio France, l'Ina et la Scam en 1993.

2 La chambre d'Echo n ${ }^{\circ}$ 1, p. 58. 
Dans le même numéro, Samy Simon, qui travaille pour le secteur de l'information, loue le documentaire, en même temps qu'il semble s'en méfier :

L'image a sur le son cet immense avantage qu'elle est le plus souvent à elle-même son propre commentaire. Le son, lui, ne peut que suggérer, et, à l'inverse de l'image, il exige de l'auditeur un effort de ses facultés d'autant plus considérables que le sujet traité est riche en matière sonore. [...] Il est certain qu'une telle symphonie acquiert, parfois, une sorte de pouvoir d'envoûtement, mais l'usage commande d'en user avec une certaine discrétion, car il est non moins certain que l'on court, autrement, le risque opposé : à savoir qu'à partir d'un certain moment, le son a perdu sa puissance d'évocation, et que, vidé de sa substance émotive, il n'est bientôt plus qu'un bruit qui se prolonge ${ }^{1}$.

Bien sûr, ces réflexions de professionnels s'inscrivent dans un contexte bien particulier. Leur comparaison entre le documentaire de radio et le documentaire de cinéma s'appuie sur un certain type de documentaire de cinéma, le documentaire d'exposition (« Expository mode ») pour reprendre le terme de Nichols (2001, p. 105), et qui consiste à faire entendre un commentaire postsynchronisé sur des images à l'origine muettes. À travers ces conceptions du documentaire de radio, on ne semble pas encore imaginer d'autre forme que celle inspirée par le documentaire d'exposition. En revanche, ces débats font déjà apparaître l'opposition chez les professionnels entre dispositif où la voix du reporter domine (le reportage), et dispositif où la matière sonore prime (le documentaire). À la fin des années 1940, Samy Simon, qui dirige l'émission Ainsi va le monde, qui dépend du journal parlé de la Radiodiffusion française, utilise le terme « documentaire » pour qualifier son émission, mais, en tant que journaliste, il signifie déjà que, si documentaire il y a dans le journalisme, il ne peut se construire qu'avec la voix d'un médiateur qui offre des repères à l'auditeur. Le son est ici décrit, en filigrane, comme une menace potentielle pour l'information. Pour éviter « le bruit qui se prolonge $»^{2}$, le journaliste s'exprimera dans le dispositif.

1 La chambre d'Echo, $\mathrm{n}^{\circ}$ 1, p. 55.

2 Idem 
En 1957, la revue Cahiers d'Études de Radio-Télévision, offre une retranscription d'une émission présentée comme un documentaire : « Sinaï », de G.B. Angioletti et S. Zavoli, qui fait partie des émissions italiennes sélectionnées pour le prix Italia. On retrouve ici les caractéristiques du documentaire de type journalistique : la voix du radioreporter, omniprésente, qui décrit ce qu'il voit, présente les interviewés, dialogue avec eux, etc. ${ }^{1} \ldots$

Peu à peu deux espaces vont s'opposer dans l'espace professionnel journalistique : d'une part, celui du studio, lieu de production des dispositifs tels que les journaux d'information, les flashs, et les papiers ; d'autre part, celui du terrain, lieu des reportages. Et même s'il est demandé au journaliste d'être polyvalent, on le classe souvent dans l'une ou l'autre des catégories ainsi créées : "présentateur » ou « reporter ». Le documentaire journalistique se situe du côté des émissions de terrain, et c'est au regard de cette opposition qu'il faut analyser la création de l'émission Vécu, sur France Inter en 1978. Michel Tauriac, qui l'a initiée, met en avant la volonté de prendre

Le contre-pied d'une des tendances de l'information radio, celle de l'analyse (produite en studio). [...] L'émission prend le parti pris de faire le moins possible de commentaires et de discours de présentation, mais avant tout de donner à voir des "faits sonores."2

$\mathrm{Si}$, pour certains journalistes, le son du terrain n'en est donc pas moins, pour d'autres, indispensable à la mise en ondes de l'information.

\section{Spécificités du documentaire journalistique}

Pour définir les spécificités du documentaire journalistique, nous avons étudié les émissions suivantes :

1 Cahiers d'Études de Radio-Télévision $\mathrm{n}^{\circ} 14$, Paris, Flammarion, $3^{\mathrm{ème}}$ trimestre 1957, pp. 164-175.

2 Propos de Michel Tauriac, cité par Guy Robert dans «Vécu : la force des images sonores ", Cahiers d'Histoire de la Radiodiffusion $n^{\circ}$ 98, Paris, Comité d'Histoire de la Radiodiffusion, octobre-décembre 2008, p. 96. 


\begin{tabular}{|l|l|}
\hline Nom de l'émission & Nom de la radio \\
\hline Ainsi va le monde & RDF, puis RTF en 1949 (1946-52) \\
\hline Le choix d'Inter & Radio France (France Inter), 1989-1999 \\
\hline Documents & RTF, 1953-56 \\
\hline Interception & Radio France (France Inter), depuis 1999 \\
\hline Le monde comme il va & RTF (Chaîne Parisienne), 1954-57 \\
\hline Vécu & Radio France (France Inter), 1978-81 \\
\hline
\end{tabular}

En nous appuyant sur l'analyse de ces émissions, nous dirons ainsi que le documentaire journalistique est un type de documentaire dont les spécificités sont les suivantes : présence de l'instance médiatique, récit linéaire, dénomination des sons, absence de flou causal, rythme saccadé, clarté des propos, standardisation des formes. Il est l'héritier du radioreportage. Il est ainsi qualifié de « reportage » dans certaines émissions. Il n'est pas forcément mis en ondes par des journalistes, mais obéit à des techniques de narration bien établies que nous définirons de la manière suivante :

Présence de l'instance médiatique : dans le documentaire journalistique, un "commentaire », le plus souvent la parole du journaliste, guide l'auditeur du début à la fin du récit. Il s'agit d'une voix qui surplombe le récit, l'ordonne. Cette voix renseigne, sur les lieux traversés, les personnes interviewées, les situations rencontrées. Elle livre des informations : chiffres, statistiques, résultats, etc.

Récit linéaire : un récit opte pour deux types de construction, thématique ou chronologique. Il est thématique s'il construit sa narration sur une succession de thèmes abordés, que l'auditeur peut aisément détacher. Il est chronologique dans la mesure où il retrace les différentes parties d'une histoire vraie, selon l'ordre dans lequel elle s'est déroulée ; il est aussi chronologique dans la mesure où il peut s'appuyer sur une construction temporelle logique (par exemple suivre un salarié durant sa journée de travail). Ces choix formels rendent le récit linéaire, ce qui favorise sa compréhension pour l'auditeur.

Dénomination des sons : l'origine des sons est précisée à l'auditeur. Chaque interviewé est présenté (fonction, statut), chaque ambiance sonore est décrite. On dira donc que les sons dans ce type de documentaire n'ont pas d'autonomie. Les sons d'ambiance ne sont jamais 
donnés à entendre sur une longue durée. L'auditeur peut les percevoir durant quelques secondes, mais très vite l'instance médiatique reprend le fil de la narration, ou fait entendre un interviewé.

L'absence du flou causal : les espaces sonores sont ici identifiés de manière claire et précise. L'auditeur sait où est enregistrée la séquence. Le documentaire de type journalistique n'a pas pour objectif de créer des univers sonores. Il se rattache au courant naturaliste, qui met la radio au service du réel qu'elle fait entendre.

Rythme saccadé : certes, aucun professionnel ne fixe de durée maximale d'un son, mais les très longues plages sonores seront ici plus rares que dans d'autres types de documentaire. Dans certaines émissions, toutes les interviews ont ainsi une durée semblable.

Clarté des propos: le documentaire journalistique poursuit une visée didactique ou informative. Il souhaite donner des informations à l'auditeur. Le récit doit donc être compréhensible, et rien ne doit nuire à la compréhension des propos. Sa valeur explicative du monde sera le critère déterminant pour évaluer sa pertinence dans le champ de la production.

Standardisation des formes: en revanche, on lui reprochera sa forme « figée », et peu évolutive. D'Ainsi va le monde en 1946, sur la RTF, à Interception, en 2010 sur France Inter, pour prendre l'exemple de deux émissions qui dépendent du secteur de l'information de la radio publique française, on remarque peu d'évolution.

Représentation de la radio : Ici la radio ne revendique pas de langage spécifique. La radio est un « support» au service du sujet traité.

Mais un genre, aussi stabilisé soit-il, subit des transformations. Quelles différences peut-on observer quand on compare toutes les émissions qui ont précédé Interception à celle-ci ? Ainsi va le Monde, Documents, Le Monde comme il va, qui sont toutes des productions médiatiques conçues par le secteur de l'information de la radio publique française ? D'une part, les récits sont toujours linéaires, et à visée informative et explicative. Mais, d'autre part, la voix de l'instance médiatique a évolué. Dans Interception, elle est en retrait par rapport à Ainsi va le Monde, Documents, et Le Monde comme il va. Le journa- 
liste ne se fixe plus comme objectif de décrire tout ce qu'il voit sur le terrain. Est-ce l'influence de la télévision? Quelques images sont certes données à entendre, mais les séquences d'expositions sont beaucoup moins longues dans Interception. Le sujet du récit a lui aussi évolué. Dans Interception, on ne trouve que rarement la référence à un « je » comme narrateur, procédé fréquent dans les émissions du journal parlé des années 1940 et 50, et qu'on retrouve dans certains ouvrages de journalistes qui ont forgé l'imaginaire de cette profession (citons, comme exemple, Au bagne, d'Albert Londres). Dans le long format radiophonique contemporain, le journaliste ne dit plus « je », comme s'il poursuivait encore sa quête d'objectivité. Le ton lui aussi a changé, et le journaliste fait preuve de moins d'éloquence, de lyrisme, et ne s'adresse plus aux interviewés comme dans les radioreportages d'avant-guerre qui, on l'a vu, nous semblent aujourd'hui artificiels. Les interventions du journaliste, qui demeurent omniprésentes dans Interception, sont diffusées avec un arrière-fond sonore (les professionnels les nomment « micros de situation »), ce qui n'était pas forcément le cas dans les années 1950, où l'on sentait assez nettement la rupture entre les séquences enregistrées sur le terrain et les interventions en studio du journaliste. Dans Interception, les micros de situation, ont pour fonction d'éviter cet effet de « rupture » entre les sons. Des premiers textes des radioreporters transmis par téléphone à des standardistes qui les écrivaient et les donnaient aux speakers qui les lisaient, aux micros de situation, le rapport entre le « terrain » et le journaliste a évolué. Pourtant, le journaliste demeure présent dans l'interaction, et le terrain ne l'a pas englouti. Un « tout sonore », d'une demi-heure, sans la présence d'un journaliste, semble aujourd'hui tout aussi inconcevable qu'hier.

Documentaire de type journalistique, Interception est donc l'ultime avatar du radioreportage d'avant-guerre. La mise en place du documentaire journalistique, tel qu' on le retrouve dans le secteur de l'information, est une tentative de codifier un genre en s'appuyant sur un dispositif qui certes peut être qualifié de " rigide », mais qui en même temps garantit sa valeur informative, en rejetant tout ce qui pourrait apparaître comme un flou sonore. Pour le secteur de l'information, la présence d'un journaliste-médiateur au sein du dispositif, la dénomination permanente des sons, et la préoccupation didactique, constituent des éléments attestant du respect des normes telles qu'elles apparaissent dans l'imaginaire journalistique : sérieux, objectivité, et rigueur. En cela, ce genre, qui s'est stabilisé depuis le milieu des 
années 1940, apparaît comme une volonté d'afficher « une identité, et un savoir-faire des journalistes ", selon l'expression de Ruellan (1993, p. 1), et contribue à légitimer le secteur de l'information.

Quelle est la place de l'auditeur au sein de ce dispositif, au regard de ces effets de sens visés? Il peut se réjouir de voir le média proposer une visée explicative du monde, à travers un dispositif qui met au centre un médiateur en qui il accorde toute sa confiance. Mais l'unicité formelle peut aussi produire d'autres effets de sens, et apparaître comme un projet de parole " techniciste » au sens de Ruellan (1993, p. 221). Ruellan associe la volonté de codifier les pratiques à une théorie fonctionnaliste du journalisme, qui vise à définir un espace propre à celui-ci, et qui rejette les pratiques amateuristes. L'auditeur pourra donc se demander si la mise en place d'un dispositif sonore identique, quel que soit le sujet traité par l'émission, ne relève pas d'une codification outrancière. Mais il faut aussi remarquer qu'Interception est une émission diffusée sur France Inter, qui est dans une logique de concurrence avec d'autres radios pour accroître son audience. L'intégration d'un médiateur (animateur ou journaliste) se retrouve dans toutes les émissions de la grille. Ce dernier élément relativise donc selon nous la critique de type techniciste, même si elle conserve sa pertinence.

Le documentaire de type journalistique poursuit une visée « informative », qui, selon Charaudeau (2005, p. 71), " consiste à faire connaître ce qui s'est passé ou ce qui est en train de se passer dans le monde de la vie sociale ». Mais cette visée nous semble avant tout être celle des émissions d'actualité, comme les journaux radiophoniques (les " news » pour reprendre le terme des professionnels. C'est pourquoi nous lui adjoindrons la visée « explicative » que Lochard et Soulages (1998, p. 120) décrivent comme complémentaire à la visée « informative », et qu'ils définissent comme « la transmission d'information et de connaissances », autour des « transformations et problématiques du monde contemporain $»$. 


\section{Références}

Charaudeau, P. (2005). Les médias et l'information. Bruxelles-Paris : De Boeck-Ina Duval, R., (1979). Histoire de la radio en France. Paris : Alain Moreau.

Lochard, G., \& Soulages, J-C. (1998). La communication audiovisuelle. Paris : Armand Colin.

Martin, M. (2005). Paquebot « Normandie », Addis-Abeba : Les premiers reportages radiophoniques au long cours. Cahiers d'Histoire de la Radiodiffusion, 84, pp. 103111.

Méadel, C. (1994). Histoire de la radio des années trente. Paris : Anthropos-Ina.

Nichols, B. (2001). Introduction to documentary. Bloominington, Indianapolis : Indiana University Press.

Prot, R. (1997). Dictionnaire de la radio. Grenoble-Bry-sur-Marne : Presses Universitaires de Grenoble-Ina.

Ruellan, D. (1993). Le professionnalisme du flou, identités et savoir-faire des journalistes français. Grenoble : Presses Universitaires de Grenoble.

Sudre, R. (1945). Le huitième art. Paris : Julliard. 